\title{
Renal, Intestinal, and Adrenal Responses to Sodium Loading in Dahl-Iwai Salt-sensitive and Salt-resistant Rats
}

\author{
Yoshio Katoh, MD, Satoshi Umemura, MD, \\ Koh-ichi Sugimoto, MD, Masakazu Tomiyama, MD, \\ Yasuhiko ABE, MD, Nobuhito HIRAwa, MD, \\ and Masao IsHII, MD
}

\section{SUMMARY}

This study compared renal and intestinal handling of sodium in DahlIwai salt-sensitive $(\mathrm{S})$ and salt-resistant $(\mathrm{R})$ rats given a normal-salt diet $(0.3 \%$ $\mathrm{NaCl})$ and a high-salt diet $(4.0 \% \mathrm{NaCl})$. Six-week-old female $\mathrm{S}$ and $\mathrm{R}$ rats $(n=7$ each) were given a normal-salt diet for 14 days followed by a high-salt diet for 3 weeks. Systolic blood pressure was significantly higher in the $S$ rats than in the $R$ rats only at the end of the high-salt diet period ( $170 \pm 5$, mean $\pm S E M$, vs $152 \pm 1 \mathrm{mmHg}, p<0.01)$. Daily sodium intake, water intake, urine volume, and urinary and fecal excretions did not significantly differ between the $R$ and the $S$ rats during the normal- and high-salt diets, except for a slight, although significant, decrease in fecal sodium excretion in the $S$ rats as compared with the $\mathbf{R}$ rats in the 2 nd week of the high-salt diet period. After switching from the normal-salt diet to the high-salt diet, urinary sodium excretion increased by 17 to 18 -fold and fecal sodium excretion increased by about 5 -fold in the 1 st week of salt loading. The changes in urinary and fecal sodium excretions did not differ significantly betweeen the groups. Cumulative sodium retention was similar in the two groups. The aldosterone/creatinine ratio in 24-hr urine, which was significantly lower in the $S$ than in the $R$ rats during the normal-salt diet, decreased to similar levels in both groups after salt loading, indicating a blunted response of aldosterone in the $\mathrm{S}$ rats. Thus, there were no discernible differences in renal and intestinal handling of sodium between the $S$ and the $R$ rats, except for a slight, but significant, difference in fecal sodium excretion in the 2nd week of the high-salt period. The results indicate that inappropriate suppression of aldosterone or some other mechanism induced by salt loading may be involved in blood pressure elevation in Dahl-Iwai S rats. (Jpn Heart J 1998; 39: 109-119)

Key words: Renal sodium handling, Intestinal sodium handling, Hypertension, Aldosterone

From the Second Department of Internal Medicine, Yokohama City Lniversity School of Medicine, Yokohama, Japan.

Address for correspondence: Satoshi Umemura, MD, Second Department of Internal Medicine, Yokohama

City University School of Medicine, 3-9 Fukuura, Kanazawa-ku, Yokohama 236, Japan.

Received for publication September 3, 1997.

Accepted October 6, 1997. 
reduced intrinsic ability of the kidney to excrete sodium and water has
been proposed as one of the pathogenic factors in primary hypertension." Membrane transport of electrolytes in the kidney and other organs, including erythrocytes and vascular smooth muscle cells, is altered in genetically hypertensive rats. ${ }^{2-4)}$ The intestinal tract and kidney have been shown to possess similar mechanisms for electrolyte transport. For instance, the mammalian colon absorbs sodium and chloride and secretes potassium and bicarbonate, and aldosterone increases the absorption of sodium in the proximal and distal portion of the rat colon. ${ }^{5)}$ Altered sodium handling in the intestine has been identified by measuring fecal sodium excretion in spontaneously hypertensive rats (SHR) given a high-salt diet. ${ }^{6-8)}$ However, little is known about sodium handling in the intestinal tract of Dahl salt-sensitive rats. Dahl salt-sensitive rats are considered one animal model of salt-induced hypertension, since the rats become hypertensive when raised on a high-salt diet.9) In this rat strain, renal cross-transplantation studies have suggested that the kidney plays a major role in the etiology of saltsensitivity and that a reduced capacity for renal sodium excretion is present before the development of hypertension..$^{9,10}$ The present study focused on intestinal sodium handling in Dahl-Iwai rats, an inbred strain of Dahl rats developed by Iwai and coworkers at Brookhaven National Laboratory, Upton, New York, USA. ${ }^{11)}$ The genetic homogeneity of Dahl-Iwai salt-sensitive (S) rats and DahlIwai salt-resistant $(R)$ rats has been confirmed by DNA fingerprint analysis. ${ }^{12)}$ In the present study, 6-week-old Dahl-Iwai $S$ and $R$ rats were given normal- and high-salt diets, and the changes in sodium intake, urine volume, urinary and fecal excretions of sodium, and urinary aldosterone excretion compared between the two groups.

\section{Materials and Methods}

Animals: Experiments were conducted in 6-week-old female Dahl-Iwai S and $\mathrm{R}$ rats $(n=7$ each) obtained from Tsukuba Research Laboratory, Eisai Co., Ltd., Tokyo, Japan.

Experimental protocols: The animals were placed individually in stainless steel metabolic cages in a room maintained under controlled light, temperature, and humidity conditions. They were given a normal-salt (standard) diet containing $0.3 \% \mathrm{NaCl}$ for 2 weeks, followed by a high-salt $(4 \% \mathrm{NaCl})$ diet for 3 weeks. The high-salt diet consisted of a pellet diet containing $68.4 \mathrm{mmol}$ sodium/100 $\mathrm{g}$ (Oriental Kobo Kogyo Co., Ltd., Tokyo, Japan). The rats were given distilled water instead of tap water during the study. While in metabolic cages, the rats had access to food and water ad libitum.

After 10 days of acclimatization in the metabolic cages, 24-hr food and 
water intake and urine volume were measured, and feces were collected from each rat. A known amount of chow was offered daily in a special trough equipped with a food trap to minimize spillage. At 24-hr intervals, food remaining in the trough or food trap was weighed, and 24-hr food consumption was calculated. Twenty-four-hour water intake was similarly determined by weighing the remaining water every day. Daily sodium intake was calculated by multiplying the sodium concentration in the chow by the amount of chow consumed. Sodium retention was determined by subtracting total sodium excreted into urine and feces from total sodium intake. The 24-hr urine and fecal samples were frozen $\left(-80^{\circ} \mathrm{C}\right)$ until further assay. To minimize the effect of daily variability, obtained data were expressed as averaged values for a normal-salt diet period (4 days) and for each week on the high-salt diet. The data obtained on the days of blood pressure measurement were deleted to eliminate potential inaccuracies due to stress imposed by blood pressure measurement. Fractional sodium retention was calculated by dividing the amount of sodium retained by the amount consumed. Fractional sodium excretions into urine and feces were also calculated by dividing sodium excreted into urine and feces by consumed sodium, respectively. Body weight was measured every week. The 24-hr water and sodium intakes, urinary volume, and the urinary and fecal excretions of sodium were all corrected by body weight. Systolic blood pressure was measured weekly by a standardized tail-cuff sphygmomanometer (UD-5000, Ueda Co., Ltd., Tokyo, Japan) after warming the animals at $37^{\circ} \mathrm{C}$ for $20 \mathrm{~min}$.

Determination of sodium content, creatinine, and aldosterone: Urinary sodium concentration was determined by flame photometry. Feces was dried completely in a microwave oven and the dry weight recorded. The feces was then ground into a powder and an aliquot $(500 \mathrm{mg})$ was wet-ashed with $2.5 \mathrm{~m} / \mathrm{of}$ concentrated $\mathrm{HNO}_{3}$ and diluted in distilled water. Sodium concentration was measured in the same manner as with urine. Daily fecal sodium excretion was calculated by multiplying the sodium concentration by the dry weight of 24-hr feces. Urinary concentrations of aldosterone and creatinine were measured by radioimmunoassay and the Folin-Wu method, respectively. The daily urinary excretion of aldosterone was expressed in terms of the aldosterone/creatinine ratio to minimize errors due to urine collection.

Statistical analysis: Measured variables are expressed as means \pm SEM. Group differences were assessed by Student's $t$ test, two-way analysis of variance (ANOVA) with repeated measures, or the nonparametric Mann-Whitney $U$ test. $P$-values of less than 0.05 were considered to indicate statistical significance. 


\section{Results}

Systolic blood pressure: Systolic blood pressure was $139 \pm 2 \mathrm{mmHg}$ in the $\mathrm{S}$ rats and $143 \pm 2 \mathrm{mmHg}$ in the $\mathrm{R}$ rats at the end of the $0.3 \% \mathrm{NaCl}$ diet period. There was no significant difference between the groups for systolic blood pressure during the normal-salt diet (Figure 1). Although systolic blood pressure also did not significantly differ between the two groups of rats in the 1st and 2nd weeks of the high-salt diet, it was significantly higher in the $S$ rats than in the $R$ rats in the 3rd week (170 \pm 5 vs. $152 \pm 1 \mathrm{mmHg}, p<0.01)$. Body weight increased in a parallel fashion in both groups during the experiment. Body weight was consistently greater in the $\mathrm{S}$ rats than in the $\mathrm{R}$ rats during both the normal-and high-salt diets (Figure 1).

Water and sodium consumption: Twenty-four-hour water and sodium intake and urine volume increased after switching from the normal-salt to high-salt diet (Table I). During the 1 st to 3 rd weeks of the high-salt diet, containing over 10 times more sodium than the normal-salt diet, dietary sodium intake rose by 11 to 15-fold in both $\mathrm{S}$ and $\mathrm{R}$ rats, while water intake increased by approximately 2fold and urine volume by 3 -fold. There were no significant differences in any of these variables between the two groups of rats.

Urinary and fecal sodium excretions: In the 1st week after transition from

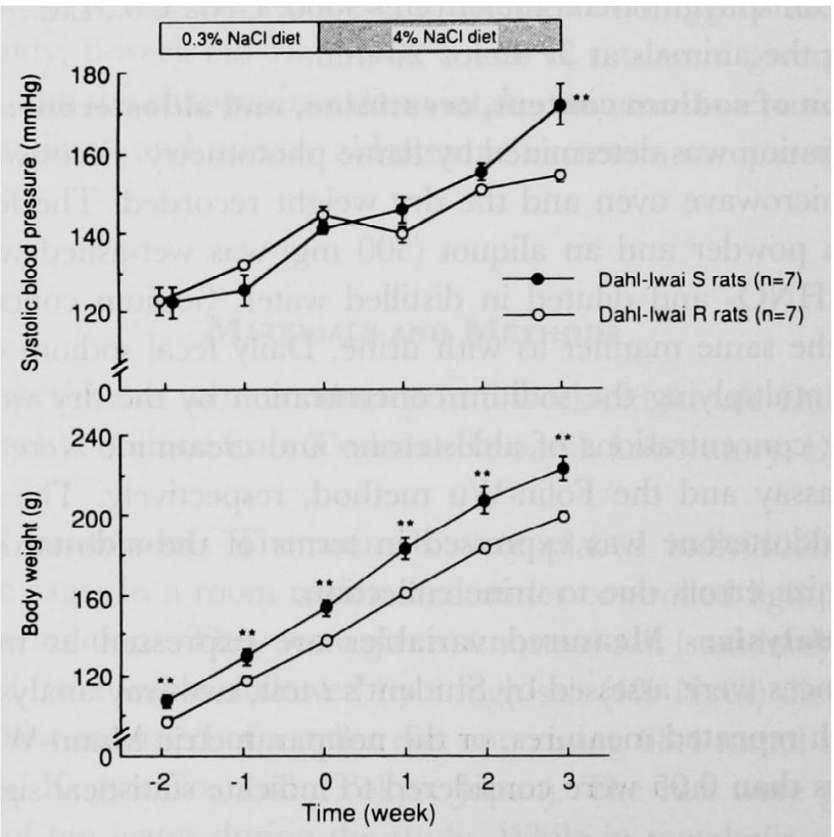

Figure 1. Line graphs show systolic blood pressure and body weight in Dahl-Iwai salt-sensitive $(\mathbf{S})$ and -resistant $(\mathbf{R})$ rats. Values are means \pm SEM. ${ }^{* *} p<0.01$ vs DahlIwai $\mathbf{R}$ rat. 
Table I. Twenty-four-hour Water Intake, Urinary Volume and Sodium Intake Normalized by Body Weight in Dahl-Iwai Salt-sensitive and -resistant Rats

\begin{tabular}{|c|c|c|c|c|c|c|}
\hline & \multicolumn{2}{|c|}{$\begin{array}{l}\text { Water intake } \\
\text { (g/day/weight) }\end{array}$} & \multicolumn{2}{|c|}{$\begin{array}{l}\text { Urine volume } \\
\text { (m//day/weight) }\end{array}$} & \multicolumn{2}{|c|}{$\begin{array}{c}\text { Sodium intake } \\
(\mu \mathrm{mol} / \mathrm{day} / \text { weight })\end{array}$} \\
\hline & $\mathbf{R}$ rats & $S$ rats & $R$ rats & $S$ rats & $\mathbf{R}$ rats & $S$ rats \\
\hline Normal-salt diet period & $0.17 \pm 0.01$ & $0.19 \pm 0.02$ & $0.08 \pm 0.00$ & $0.07 \pm 0.01$ & $5.45 \pm 0.29$ & $5.26 \pm 0.08$ \\
\hline High-salt 1st week & $0.37 \pm 0.02$ & $0.38 \pm 0.01$ & $0.23 \pm 0.01$ & $0.25 \pm 0.01$ & $73.27 \pm 0.92$ & $72.21 \pm 0.68$ \\
\hline 2nd week & $0.32 \pm 0.02$ & $0.31 \pm 0.01$ & $0.21 \pm 0.02$ & $0.22 \pm 0.01$ & $65.64 \pm 0.99$ & $63.37 \pm 0.77$ \\
\hline 3rd week & $0.27 \pm 0.02$ & $0.28 \pm 0.01$ & $0.17 \pm 0.01$ & $0.19 \pm 0.01$ & $57.56 \pm 0.42$ & $58.04 \pm 0.73$ \\
\hline
\end{tabular}

$\mathrm{R}=$ Dahl-Iwai salt-resistant; $\mathrm{S}=\mathrm{Dah}$ l-Iwai salt-sensitive. Values are means $\pm \mathrm{SEM}$.

Table II. Twenty-four-hour Sodium Excretion into Urine and Feces Normalized by Body Weight in Dahl-Iwai Salt-sensitive and -resistant Rats

\begin{tabular}{|c|c|c|c|c|}
\hline & \multicolumn{2}{|c|}{$\begin{array}{l}\text { Urinary sodium excretion } \\
(\mu \mathrm{mol} / \text { day } / \text { weight })\end{array}$} & \multicolumn{2}{|c|}{$\begin{array}{l}\text { Fecal sodium excretion } \\
(\mu \mathrm{mol} / \text { day } / \text { weight })\end{array}$} \\
\hline & $\mathrm{R}$ rats & $S$ rats & $\mathbf{R}$ rats & $S$ rats \\
\hline Normal-salt diet period & $3.05 \pm 0.06$ & $3.27 \pm 0.13$ & $0.94 \pm 0.04$ & $0.84 \pm 0.03$ \\
\hline High-salt lst week & $56.21 \pm 1.18$ & $56.39 \pm 1.09$ & $4.50 \pm 0.30$ & $4.04 \pm 0.18$ \\
\hline 2nd week & $54.13 \pm 1.14$ & $52.58 \pm 0.65$ & $3.94 \pm 0.29$ & $3.12 \pm 0.16^{*}$ \\
\hline 3rd week & $45.84 \pm 0.98$ & $46.76 \pm 0.72$ & $3.42 \pm 0.19$ & $3.30 \pm 0.20$ \\
\hline
\end{tabular}

$\mathrm{R}=$ Dahl-Iwai salt-resistant; $\mathrm{S}=$ Dahl-Iwai salt-sensitive. Values are means $\pm \mathrm{SEM}$. ${ }^{*} p<0.05$ vs. $\mathrm{R}$ rats.

the normal- to the high-salt diet, urinary sodium excretion increased by 17- to 18-fold in both groups, while fecal sodium excretion increased by only about 5 fold (Table II). The urinary and fecal sodium excretions tended to decrease in the $\mathrm{S}$ and the $\mathrm{R}$ rats thereafter. There were no significant differences in urinary sodium excretion between the two groups during the normal- and high-salt diets. Fecal sodium excretion was significantly lower in the $S$ rats than in the $R$ rats in the 2 nd week of the high-salt diet, but did not differ significantly between the groups at any other time.

Fractional sodium excretion and sodium retention: After switching to the high-salt diet, fractional sodium excretion into urine increased similarly in the $\mathrm{S}$ and $\mathrm{R}$ rats, whereas fractional sodium excretion via the feces fell to one-third of that during the normal-salt diet in both strains of rat (Table III). Fractional sodium retention decreased in both groups when the animals were switched to the high-salt from the normal-salt diet (Table III). During the high-salt diet, fractional sodium retention and fractional sodium excretions into urine and feces were similar in both groups, although fractional sodium excretion into feces in the $S$ rats tended to decrease during the 2nd week of the high-salt diet. The cumulative sodium balance was calculated during the high-salt diet. It did not differ significantly between the $\mathrm{S}$ and the $\mathrm{R}$ rats (Figure 2).

Urinary aldosterone excretion: The daily urinary excretion of aldosterone 
Table III. Fractional Sodium Retention and Fractional Sodium Excretion into Urine and Feces in Dahl-Iwai Salt-sensitive and -registant Rats

\begin{tabular}{ccccccccc}
\hline & \multicolumn{2}{c}{ Retention, $\%$} & & \multicolumn{2}{c}{ Urinary excretion, \% } & & \multicolumn{2}{c}{ Fecal excretion, \% } \\
\cline { 2 - 3 } & R rats & S rats & & R rats & S rats & & R rats & S rats \\
\hline Normal-salt diet period & $24.9 \pm 2.7$ & $21.5 \pm 2.3$ & & $57.4 \pm 2.6$ & $62.4 \pm 2.2$ & & $17.7 \pm 0.6$ & $16.1 \pm 0.6$ \\
High-salt 1st week & $16.9 \pm 1.2$ & $16.1 \pm 1.0$ & $77.0 \pm 1.3$ & $78.3 \pm 1.1$ & & $6.1 \pm 0.4$ & $5.6 \pm 0.3$ \\
2nd week & $11.4 \pm 1.6$ & $11.9 \pm 0.7$ & & $82.6 \pm 1.7$ & $83.2 \pm 0.9$ & & $6.0 \pm 0.4$ & $4.9 \pm 0.3^{\dagger}$ \\
3rd week & $14.2 \pm 1.3$ & $15.5 \pm 2.0$ & & $79.8 \pm 1.2$ & $78.7 \pm 1.9$ & & $5.9 \pm 0.3$ & $5.8 \pm 0.4$ \\
\hline
\end{tabular}

$\mathrm{R}=$ Dahl-Iwai salt-resistant; $\mathrm{S}=$ Dahl-Iwai salt-sensitive. Values are means $\pm \mathrm{SEM} .{ }^{\dagger} p<0.08$ vs. $\mathrm{R}$ rats.

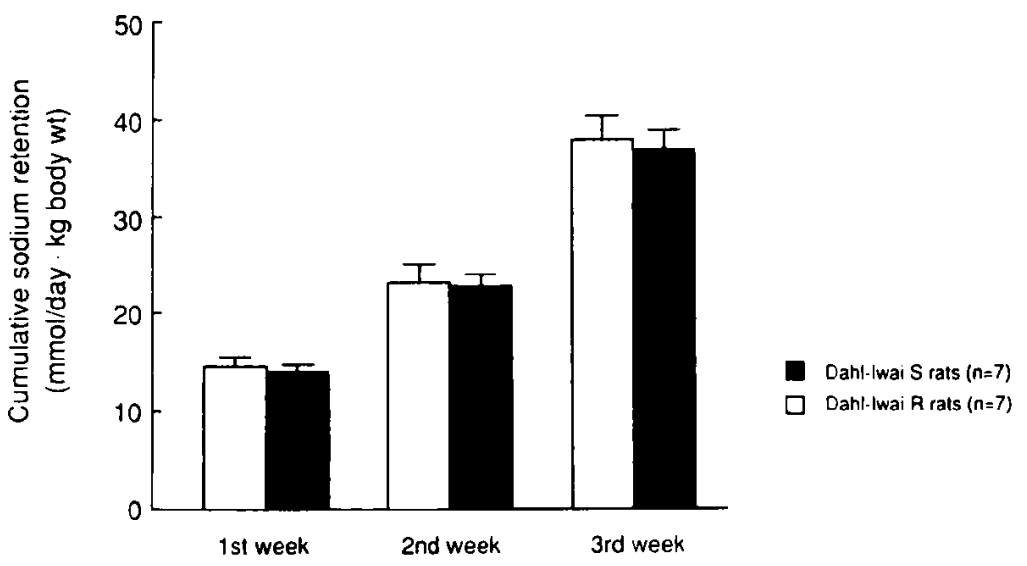

Figure 2. Columns show cumulative sodium retention in Dahl-Iwai salt-sensitive (S) and -resistant $(R)$ rats. No significant differences were found between the two groups during the high-salt diet.

Table IV. Urinary Aldosterone/Creatinine Ratio in Dahl-Iwai Salt-sensitive and -resistant Rats

\begin{tabular}{ccc}
\hline & Aldosterone/creatinine ratio $\left(\times 10^{-7}\right)$ & \\
\cline { 2 - 3 } & R rats & S rats \\
\hline Normal salt diet period & $20.5 \pm 1.2$ & $15.0 \pm 0.9^{* *}$ \\
1st week & $5.0 \pm 0.4$ & $5.2 \pm 0.6$ \\
High salt 2nd week & $7.4 \pm 0.7$ & $8.3 \pm 0.3$ \\
3rd week & $6.7 \pm 0.4$ & $6.5 \pm 0.7$ \\
\hline
\end{tabular}

$\mathrm{R}=$ Dahl-Iwai salt-resistant; $\mathrm{S}=$ Dahl-Iwai salt-sensitive. Values are means $\pm \mathrm{SEM} .{ }^{* *} p<0.01 \mathrm{vs.} \mathrm{R}$ rats.

was expressed in terms of the aldosterone/creatinine ratio in urine samples collected over $24 \mathrm{hr}$. The ratio was significantly greater in the $\mathrm{R}$ rats than in the $\mathrm{S}$ rats during the normal-salt diet. However, the ratio rapidly decreased to similar levels in both groups after the high-salt diet was started (Table IV). When the changes in the aldosterone/creatinine ratio were compared between the two groups, the reduction in the ratio was significantly smaller in the $S$ rats than in the $\mathrm{R}$ rats during all 3 weeks of the high-salt diet (Figure 3). 


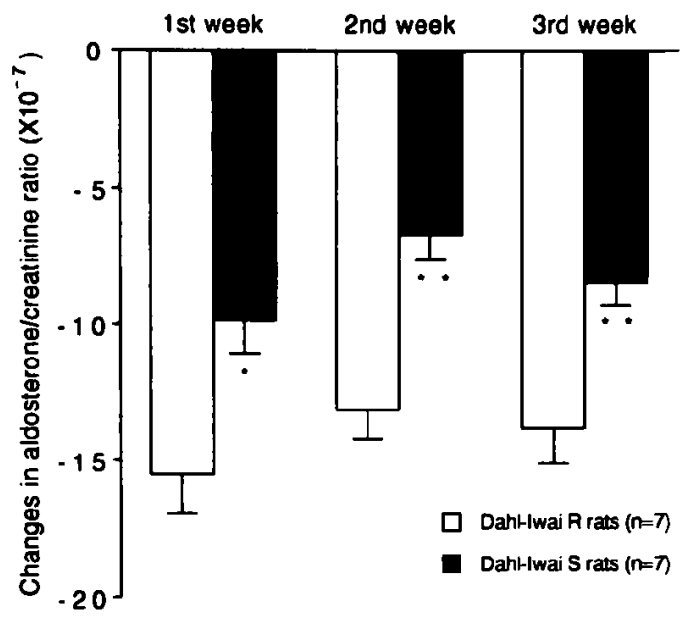

Figure 3. Columns show the changes in urinary aldosterone/creatinine ratio during the high-salt diet. The reduction in the ratio was significantly greater in the $\mathrm{S}$ rats than in the $\mathrm{R}$ rats throughout salt loading. ${ }^{*} p<0.05,{ }^{* *} p<0.01$ vs. Dahl-Iwai $\mathrm{R}$ rat.

\section{Discussion}

Systolic blood pressure gradually increased in both $\mathrm{R}$ and $\mathrm{S}$ rats after switching from a normal-salt to a high-salt diet and was significantly higher in the $\mathrm{S}$ rats after 3 weeks of salt loading. There were no significant differences in water intake, urine volume, sodium intake or urinary and fecal excretions of sodium between the $\mathrm{R}$ and $\mathrm{S}$ rats during the normal-salt diet. Although sodium intake increased by 13 - to 14-fold, urinary sodium excretion by 17 - to 18 -fold, and fecal sodium excretion by 5 -fold in both the $\mathrm{R}$ and $\mathrm{S}$ rats in the 1 st week of the highsalt diet, these changes also did not differ significantly between the two groups of rats. Even when sodium balance was expressed in terms of fractional retention or the fractional urinary and fecal excretions of sodium, there were no differences between the groups in any of these variables during the 1st week of the high-salt diet. Thus, we found no evidence that prehypertensive Dahl-Iwai $\mathrm{S}$ rats retained more sodium than did Dahl-Iwai $R$ rats when they were transferred from a normal- to a high-salt diet. Cumulative sodium retention also did not differ between Dahl-Iwai $\mathbf{S}$ and $\mathbf{R}$ rats after initiation of the high-salt diet. Our results are consistent with those of previous studies demonstrating that net sodium balances do not differ between Dahl salt-resistant rats and prehypertensive Dahl salt-sensitive rats exposed to a high-salt diet. ${ }^{13.14)}$ Roman and Osborn ${ }^{13)}$ observed a transient elevation of extracellular fluid volume in Dahl salt-sensitive rats on the 1st day of a high-salt diet, but not thereafter. Overbeck et al. ${ }^{15)}$ reported that plasma volume, but not extracellular fluid volume, is expanded in hypertensive Dahl salt-sensitive rats fed a high-salt diet. It is conceivable that a redistribution 
of extracellular fluid into an intravascular compartment may elevate blood pressure, even in the absence of a net increase in sodium balance. However, we were unable to address the possibility that an alteration in extracellular or plasma volume may have been related to the rise in blood pressure in salt-fed Dahl-Iwai $S$ rats in the present study. Even if the change in extracellular volume is similar in the $\mathrm{S}$ and $\mathrm{R}$ rats, differences in the production of endogenous vasoactive factors, such as an endogenous digitalis-like factor that is released by salt loading, or altered production of vasodilating prostaglandins may have been involved in the blood pressure elevation in the $\mathrm{S}$ rats. ${ }^{16,177}$

Studies have shown that a reduced capacity of the kidney to excrete sodium plays a major role in the development of hypertension in Dahl salt-sensitive rats, with a shift of the pressure-natriuresis relation toward higher pressure levels. This abnormal renal handling of sodium may be due to increased reabsorption of sodium at the proximal tubule ${ }^{18)}$ or to abnormal renal hemodynamics, ${ }^{19)}$ In the present study, urinary sodium excretion and fractional sodium excretion did not differ significantly between the $S$ and the $R$ rats during the 3rd week of the highsalt diet, when systolic blood pressure was significantly higher in the former. This finding suggests an abnormal pressure-natriuresis relationship in the $\mathrm{S}$ rats. ${ }^{19,20)}$

Despite elaborate measurement of water and sodium balance, the present study provided no evidence supporting the putative retention of sodium in the $\mathrm{S}$ rats during high salt intake. It did, however, demonstrate that fecal sodium excretion also increased in both $\mathrm{R}$ and $\mathrm{S}$ rats, albeit to a lesser extent than urinary sodium excretion, in response to salt loading. Earlier studies have suggested that the gastrointestinal tract has an important part in the maintenance of electrolyte homeostasis. ${ }^{16-18)}$ The proximal and distal colon absorbs sodium by several active transport processes in rats and humans. ${ }^{21-23)}$ The fecal excretion of sodium is similar in SHR and Wistar-Kyoto rats (WKY) receiving a low-sodium diet, ${ }^{7}$ while it increases to a greater extent in SHR than in WKY receiving a high-salt diet. ${ }^{6,8}$ In the present study, fecal sodium excretion was significantly lower in the $\mathrm{S}$ rats than in the $\mathrm{R}$ rats only in the 2 nd week of the high-salt diet, and fractional fecal excretion of sodium showed a similar tendency. Thus, we speculate that the decreased fecal sodium excretion in the $\mathrm{S}$ rats during the 2 nd week of the highsalt diet may be involved in the blood pressure elevation observed in the 3rd week of the high-salt diet. However, the change in fecal sodium excretion was not so large as to affect the sodium retention in the $\mathrm{S}$ rats. The patho-physiological implications of these findings remain to be confirmed in further studies.

It has been reported that the renin-angiotensin-aldosterone system is suppressed in Dahl salt-sensitive rats ${ }^{24,25)}$ and that the lowered plasma level of aldosterone is due to decreased plasma renin activity ${ }^{24)}$ or to some other causes. ${ }^{25)}$ Consistent with these reports, the urinary aldosterone/creatinine ratio in 24-hr 
urine samples was significantly lower in the $\mathrm{S}$ rats than in the $\mathrm{R}$ rats before salt loading. However, since PRA was not measured in the present study, it is unclear whether or not the lower urinary aldosterone/creatinine ratio in the $\mathrm{S}$ rats was due to a decrease in PRA. Interestingly, in the present study the urinary aldosterone/creatinine ratio decreased rapidly to similar levels in both $S$ and $R$ rats after transition from the normal-salt diet to the high-salt diet. The decrease in the ratio was significantly smaller in the $S$ rats than in the $R$ rats throughout the high salt diet period, suggesting that the aldosterone response to salt loading was blunted in the $\mathrm{S}$ rats. Rodriguez-Sargent et $a ._{.}{ }^{26}$ demonstrated that the plasma concentration of aldosterone is lower in Dahl $\mathrm{S}$ rats than in $\mathrm{R}$ rats during both low-salt and normal-salt diets, and that the changes in plasma aldosterone levels associated with alterations in salt intake were similar in both rats. Thus, the results of our study seem to be in conflict with theirs. Prior studies have shown that although aldosterone synthase catalyzes the conversion of 11-deoxycorticosterone to aldosterone at a slower rate in Dahl $S$ rats than in $\mathrm{R}$ rats, these strains differ with respect to the transcriptional regulation or message stability, or both, of aldosterone synthase, possibly resulting in elevation of the plasma aldosterone/renin ratio in the former. ${ }^{27)}$ It is also known that sodium restriction specifically increases the late pathway of aldosterone biosynthesis, where corticosterone is converted to aldosterone, in rats. ${ }^{28\rangle}$ It is not known, however, how continuous salt loading modifies the activity of enzymes involved in the synthesis and release of aldosterone in Dahl-Iwai $S$ and $R$ rats. Delineation of the mechanism underlying the blunted response of aldosterone to salt loading in the present study must await the results of further investigations.

Aldosterone, the most potent mineralocorticoid secreted by the adrenal gland, stimulates renal sodium reabsorption in the distal tubule and cortical collecting duct. Aldosterone increases the absorption of sodium by different mechanisms in the proximal and distal portion of the rat colon, i.e., it stimulates electrogenic sodium transport by inducing amiloride-sensitive sodium channels in the apical membrane in the proximal colon, whereas it increases sodium absorption without inducing amiloride sensitivity or altering transmembrane potential difference in the distal colon. ${ }^{22,29)}$ The present study showed that a 13-fold increase in sodium intake enhanced the intestinal excretion of sodium by 5 -fold, although this was less than the increment in renal sodium excretion. The changes in fecal sodium excretion in the $S$ rats and the $R$ rats seem to have been due partly to reduced production of aldosterone. As mentioned above, an abnormal pressure-natriuresis relationship was present in the $\mathrm{S}$ rats. This abnormal relationship seems to have been partly related to inappropriate suppression of aldosterone. Furthermore, aldosterone has been reported to redistribute extracellular fluid volume and thereby increase plasma volume in nephrectomized rats. ${ }^{30}$ 
Although the reasons remain unclear, a blunted response of aldosterone to salt loading, resulting in impediment of pressure natriuresis, modification of fecal sodium excretion, and redistributuion of extracellular volume, appears to be involved in the elevation of blood pressure in Dahl-Iwai salt-sensitive rats during salt loading.

\section{REFERENCES}

1. Guyton AC, Cleman TG, Cowley AW, Scheel KW, Manning RD Jr, Norman RA Jr. Arterial pressure regulation: overriding dominance of the kidneys in long term-regulation and in hypertension. Am J Med 1972; 52: 584-94.

2. Postnov YU, Orlov S, Gulak P, Shevchenco A. Altered permeability of the erythrocyte and potassium ions in spontaneously hypertensive rats. Pflügers Arch 1976; 365: 257-63.

3. Jones AW. Altered ion transport in vascular smooth muscle from spontaneously hypertensive rats. Circ Res 1973; 33: 563-72.

4. Jones AW. Altered transport in large and small arteries from spontaneous hypertensive rats and the influence of calcium. Circ Res 1974; 34-35 (Suppl 1): 117-22.

5. Hirsch D, Pace P, Binder HJ, Haysllett JP. Evidence that aldosterone influences transport in target tissues by dissimilar mechanisms. Am J Physiol 1985; 248: F507-F512.

6. Lundin S, Herlitz H, Hallbäck-Nordlander M, Ricksten SE, Göthber G, Berglund G. Sodium balance during development of hypertension in spontaneously hypertensive rat (SHR). Acta Physiol Scand 1982; 115: 317-23.

7. Beierwaltes WH, Arendshorst WJ, Klemmer PJ. Electrolyte and water balance in young spontaneously hypertensive rats. Hypertension 1982; 4: 908-15.

8. Lubcke R, Barbezat GO. Intestinal ion transport in rats with spontaneous arterial hypertension. Clin Sci 1988; 75: 127-33.

9. Dahl LK, Heine M, Tassinari L. Effect of chronic excess salt ingestion: evidence that genetic factors play an important role in susceptibility to experimental hypertension. J Exp Med 1962; 115: 1173-90.

10. Dahl LK, Heine M1, Thompson K. Genetic influence of the kidneys on blood pressure, evidence from chronic renal homografts in rats with opposite predispositions to hypertension. Circ Res 1974; 34: 94101 .

11. Iwai J, Heine M. Dahl salt-sensitive rats and human essential hypertension. J Hypertens 1986; 4 (Suppl 3): S29-S31.

12. Yamaguchi $\mathrm{S}$, Umemura $\mathrm{S}$, Iwamoto $\mathrm{T}$, et al. DNA fingerprint analysis of Dahl-Iwai salt-sensitive rats (S) and salt-resistant rats (R). Life Sci 1994; 55; 701-5.

13. Roman RJ, Osborn JL. Renal function and sodium balance in conscious Dahl $\mathbf{S}$ and $\mathbf{R}$ rats. Am $\mathbf{J}$ Physiol 1987; 252: R833-R841.

14. Genain CP, Reddy SR, Ott CE, Van Loon GR, Kotchen TA. Failure of salt loading to inhibit tissue norepinephrine turnover in prehypertensive Dahl salt-sensitive rats. Hypertension 1988; 12: 568-73.

15. Overbeck HW, Ku DD, Rapp JP. Sodium pump activity in arteries of Dahl salt-sensitive rats. Hypertension 1981; 3: 306-12.

16. DeWander HE, MacGregor G. Dahl's hypothesis that a saluretic substance may be responsible for a sustained rise in arterial pressure: its possible role in essential hypertension. Kidney Int 1980; 18: 1-9.

17. Uehara $Y$, Tobian L, Iwai J, Ishii M, Sugimoto $T$. Alterations of vascular prostacyclin and thromboxane $A_{2}$ in Dahl genetical strain susceptible to salt-induced hypertension. Prostaglandins 1987; 33: 72738.

18. Roos JC, Kirchner KA, Abernethy JD, Langford HG. Differential effect of salt-loading on sodium and lithium excretion in Dahl salt-resistant and -sensitive rats. Hypertension 1984; 6: 420-4.

19. Tobian L, Lange J, Azar S, et al. Reduction of natriuretic capacity and renin release in isolated, bloodperfused kidneys of Dahl hypertension-prone rats. Circ Res 1978; 43 (Suppl I): I-92-I-97.

20. Roman RJ. Abnormal renal hemodynamics and pressure-natriuresis relationship in Dahl salt-sensitive 
rats. Am J Physiol 1986; 251: F57-F65.

21. Sellin JH, De Soignie R. Ion transport in human colon in vitro. Gastroenterology 1987; 93: 441-8.

22. Foster ES, Bundinger ME, Haysllett JP, Binder HJ. Ion transport in proximal colon of the rat: sodium depletion stimulates neutral sodium chloride absorption. J Clin Invest 1986; 77: 228-35.

23. Binder HJ, Foster ES, Bundinger ME, Haysllett JP. Mechanism of electroneutral sodium chloride absorption in distal colon of the rat. Gastroenterology 1987; 93: 449-55.

24. Rapp JP, Tan SY, Margolius HS. Plasma mineral corticoids, plasma renin, and urinary kallikrein in salt-sensitive and salt resistant rats. Endocrine Res Comm 1987; 5: 35-41.

25. Kusano E, Baba K, Rapp J, et al. Adrenal renin in Dahl salt-sensitive rats: a genetic study. J Hypertens 1986; 4 (Suppl 5): 20-2.

26. Rodriquez-Sargent C, Cangiano JL, Opava-Stitzer S, Martinez-Maldonado M. Renal $\mathrm{Na}^{+}, \mathrm{K}^{+}$-ATPase in Okamoto and Dahl hypertensive rats. Hypertension 1981; 3(Suppl II): II-86-II-91.

27. Cover CM, Wang JM, Lezin ES, et al. Molecular variants in the P450cllAS gene as determinants of aldosterone synthase activity in the Dahl rat model of hypertension. J Biol Chem 1995; 270: 16555-60.

28. Adler GK, Chen R, Menachery AI, et al. Sodium restriction increases aldosterone biosynthesis by increasing late pathway, but not early pathway, messenger ribonucleic acid levels and enzyme activty in normotensive rats. Endocrinology 1993; 133: 2235-40.

29. Foster ES, Zimmerman TW, Haysllett JP, Binder HJ. Corticosteroid alteration of active electrolyte transport in rat distal colon. Am J Physiol 1983; 245: G668-G675.

30. Ishii M, Yamakado M, Murao S. The extrarenal effects of aldosterone on the distribution of extracellular fluid in conscious adrenalectomized-nephrectomized rats. Pflügers Archiv 1985; 404: 273-7. 